\title{
Discours de la crise, crise du discours
}

\section{Stéphane Longuet et Jaime Marques Pereira}

\section{(2) OpenEdition \\ Journals}

Édition électronique

URL : http://journals.openedition.org/ei/971

DOI : 10.4000/ei.971

ISSN : 2553-1891

\section{Éditeur}

Association Économie et Institutions

\section{Référence électronique}

Stéphane Longuet et Jaime Marques Pereira, «Discours de la crise, crise du discours », Économie et institutions [En ligne], 22 | 2015, mis en ligne le 01 juin 2015, consulté le 10 décembre 2020. URL http://journals.openedition.org/ei/971 ; DOI : https://doi.org/10.4000/ei.971

Ce document a été généré automatiquement le 10 décembre 2020.

Revue Économie et institutions 


\title{
Discours de la crise, crise du discours
}

\author{
Stéphane Longuet et Jaime Marques Pereira
}

Nous remercions l'ensemble des participants au colloque "Crise de l'euro, effets discursifs et changements institutionnels : approches transdisciplinaires » (3-5 décembre 2014, Amiens) pour la richesse de leurs contributions aux discussions. Nous remercions en particulier Odile LakomskiLaguerre pour ses relectures attentives de ce texte. Nous restons évidemment seuls responsables des erreurs ou omissions qu'il contient.

1 À la différence de ce qui a été le cas dans les autres disciplines des sciences humaines et sociales, le tournant cognitif (Orléan, 2002) en économie ne s'est pas accompagné d'une interrogation systématique sur le rôle et les fonctions du langage. Une telle situation est pourtant surprenante. Après tout, à un niveau fondamental les activités économiques reposent au moins implicitement sur l'utilisation du langage : comment marchander sans échanger des mots, propositions et contrepropositions etc. ? Comment faire fonctionner une organisation sans donner des ordres, des conseils ou tenter de convaincre les partenaires? Comment opérer une répartition de ressources sans en justifier, par le langage, le bien-fondé ? L'analyse du langage en économie semble avoir aujourd'hui une position comparable à celle qu'avait le concept d'institution à l'aube du renouveau institutionnaliste il y a une trentaine d'année : implicitement présent, il ne constitue pas, d'un point de vue théorique un objet central d'analyse.

2 Le langage est cependant devenu, d'un point de vue empirique, au centre de certaines préoccupations. La question de la communication des banques centrales par exemple constitue un terrain propice à l'étude des effets des mots, des arguments et de tout ce que l'on peut ranger sous l'expression de "discours des banques centrales". De même, l'importance des articles de presse, financiers ou autres, ou celle des discours managériaux, constituent autant de phénomènes empiriques révélant que, comme la monnaie, comme les règles et les institutions, le langage n'est pas neutre et que, produisant des effets sur les activités et valeurs économiques, il doit être considéré comme l'un des objets de l'économie politique. 
3 C'est sans doute autour de la notion de discours que s'articulent, en économie comme ailleurs, les recherches s'intéressant aux effets du langage. B. Maris avait dirigé, en 2002, un numéro de Sciences de la société sur les discours de l'économie avec comme objectif de présenter des textes traduisant, à la suite de McCloskey (1983), les différentes manifestations d'une rhétorique économique c'est-à-dire les modes d'argumentation utilisés pour emporter la conviction (Maris, 2002a et b). Plus largement, un discours économique peut renvoyer à toute production langagière portant sur les phénomènes économiques que ces productions soient, ou non, le fait d'économistes professionnels. Ainsi l'analyse des discours économiques sera pluridisciplinaire, associant sociologues et analystes du langages (Temmar, Angermuller, Lebaron, 2013).

4 La notion de discours est polysémique, comme cela a été maintes fois souligné. Si, en première approximation, un discours renvoie à un ensemble d'énoncés au-dessus de la phrase, il doit être considéré non pas comme un objet figé mais comme un "mode d'appréhension du langage» (Maingueneau, 1995, p.6). Maingueneau (2009) met en évidence les « idées forces » associées au discours. Le discours, souligne-t-il est orienté et lié à la communauté dans laquelle il a été énoncé ; il est contextualisé, ce qui signifie que son sens ne peut être saisi que dans son contexte d'énonciation alors même qu'il peut contribuer à le modifier ; et enfin, qu'il constitue une forme d'action. On pourrait alors considérer qu'une analyse des discours économiques devrait articuler plusieurs approches : les analystes du langage étudient la production langagière en elle-même et ne s'attachent donc pas à une analyse du contenu (Guilbert, 2011, p. 25); la sociologie se concentre sur les conditions et effets sociaux et institutionnels des discours, étudiant par exemple la production et la diffusion des discours économiques (Lebaron, 2013) et l'économie intègre une analyse des caractéristiques et limites des énoncés et théories économiques.

5 Les analyses de discours, d'ores et déjà présentes dans certaines approches économiques, manifestent ainsi la richesse potentielle de telles études. Notre interrogation portera ici sur les conditions qui permettent de les intégrer de manière plus systématique dans un cadre d'analyse d'économie politique. Il s'agira d'esquisser les éléments de ce que pourrait être une économie politique des discours. En utilisant le terme «économie politique » nous voulons marquer notre volonté de ne pas tenir pour acquis la pertinence des outils et concepts de ce qu'on appelle «science économique». Nous voulons aussi souligner une démarche qui envisage la discipline économique comme l'une des branches des sciences sociales, dont elle considère pouvoir utilement reprendre certains concepts et certaines méthodes dans une relation de fécondation croisée, éloignée de cet impérialisme méthodologique pratiqué parfois par les économistes.

Dans un premier temps nous essaierons de repérer les aspects qui peuvent constituer les éléments d'une économie politique des discours. Nous verrons en particulier en quoi la question des conditions de production et de diffusion des discours conduit à une interrogation sur la question des idéologies. Les moments de crises apparaîtront alors significatifs dans la mesure où s'y expriment à la fois la volonté d'agir sur le contexte économique par les actes et par les mots et la volonté de maintenir ou de recomposer les discours correspondant à une représentation dominante de l'économie et de la société. 


\section{Intégrer le discours et son analyse à l'économie politique}

7 Quelle peut être la place de l'analyse du discours au sein de l'économie politique ? Nous commencerons par distinguer deux grands types d'intégration du langage en confrontant le wording pratiqué pour étudier la communication des banques centrales par exemple à une intégration du discours comme activité sociale qui renvoie plus particulièrement aux approches régulationnistes ou conventionnalistes (1.1). La compréhension des effets des discours exige alors d'intégrer les conditions sociales et institutionnelles de leur production et de leur diffusion (1.2).

\subsection{Relations économiques et langage : quelques pistes}

Prenant pour point de départ les analyses de la communication des banques centrales, nous soulignerons que les discours économiques ne peuvent être abordés à partir de la seule théorie de l'information, mais doivent intégrer l'activité d'interprétation (a). Les théories des discours nous permettront de saisir en quoi, au-delà de la diversité des approches, une telle activité peut être saisie en combinant démarches quantitatives et qualitatives. C'est en abordant les discours comme activités sociales que l'on peut saisir comment ils enrichissent ou sont susceptibles d'enrichir certaines approches d'économie politique comme l'économie des conventions ou la théorie de la régulation (b).

9 a) Les travaux sur la communication des banques centrales peuvent être considérés comme une prise en compte des discours des acteurs économiques. La communication est cependant pensée, non pas en relation avec l'activité sociale du discours mais avec une théorie de l'information. La question posée est en effet celle des effets positifs ou négatifs des signaux envoyés. Ainsi dans Blinder et al. (2008) l'activité de communication est envisagée à travers une grille signal/bruit : le signal peut orienter les anticipations et les coordonner mais risque d'accroître la volatilité du prix des actifs. En termes de langage, l'unité adaptée à l'appréhension du signal est le mot et le wording est défini comme la correspondance entre un ensemble de mots et un ensemble de variables mesurant l'activité économique et financière. Il s'agit d'une méthode d'autant plus adaptée qu'elle permet d'utiliser certains des outils traditionnels de l'analyse économique à savoir l'économétrie.

Or comme le montre l'article d'E. Carré (ce numéro), le wording comme analyse purement empirique est insuffisant : le sens des mots ne peut se saisir que replacé dans le contexte théorique au sein duquel ils trouvent leur origine. Le wording peut alors être utile comme un signe impliquant la mise en relation avec un contexte théorique. La cohérence de la théorie conduit alors à mettre en évidence la potentialité des mots et leurs contenus implicites. Plus qu'un simple exercice économétrique, le wording apparaît comme la mise en œuvre d'un processus d'interprétation, c'est-à-dire comme la première étape d'une herméneutique économique. Ainsi, par exemple, dans le cadre d'une nouvelle synthèse néoclassique reliant nouvelle macroéconomie classique (NEC) et nouvelle économie keynésienne (NEK), le repérage de l'utilisation de certains mots permet d'identifier le moment pendant lequel la politique monétaire utilisera une communication proche de la nouvelle macroéconomie classique ou de la nouvelle économie keynésienne. 
11 Cette herméneutique permet en outre d'identifier les caractéristiques de la multiplicité des effets des discours. Ceux-ci d'adressent à des publics différents pour lesquelles ces mêmes mots, énoncés ou discours auront des significations différentes. Prenons par exemple le syntagme "réformes structurelles ». Si l'on classe la population en sousensembles délimités selon le degré plus ou moins important de connaissance de la théorie économique, cela introduit à une différence de perception du même terme. Pour les noninformés, le terme peut évoquer de manière positive le mouvement par rapport à l'immobilisme (« réformes»), la profondeur par rapport à la superficialité («de structures »). Pour un second ensemble avec un référentiel théorique plus large, le terme sera associé au couple flexibilité/rigidité. Enfin pour un troisième ensemble, il sera lié aux concepts de NAIRU, au taux de chômage naturel et à l'idée que, sous conditions d'information parfaite et d'anticipations rationnelles, les politiques anticipées, qu'elles soient budgétaires ou monétaires seront inefficaces, seules la flexibilisation des marchés et la diffusion de l'information étant susceptibles de faire baisser le taux de chômage naturel ou d'accélérer le taux de croissance naturel de l'économie.

On pourrait alors ajouter un dernier sous ensemble, composé d'une partie des économistes professionnels qui ont la conscience des présupposés théoriques et des limites des approches qui sous-tendent l'idée de « réformes structurelles ». L'un des plus important de ces présupposés étant que la stabilité des marchés, n'ayant pu être démontrée au sein même de la théorie économique dominante ${ }^{1}$, la défense de la flexibilité, même soutenue par des modèles sophistiqués, apparaît comme relevant plus de croyances économiques que d'un discours scientifique. Au-delà des économistes se référant à des traditions critiques (marxistes, keynésiens, institutionnalistes), un ensemble non négligeable d'auteurs, tout en voulant rester proches du cadre d'analyse dominant, souhaitent tirer toutes les conséquences des limites théoriques de la théorie économique néoclassique. C'est le cas, par exemple, du courant dit "post-walrasien » (Colander, ed 1996) qui, tout en récusant une quelconque référence "hétérodoxe» (Colander, 2003), et en réaffirmant la nécessité d'une analyse économique comme modélisation, étudie toutes les caractéristiques et conséquences des problèmes de coordination évacués par la plupart des modèles économiques dominants.

13 Toutes ces opérations critiques, qu'elles proviennent d'acteurs proches ou éloignés de la théorie dominante, constituent des opérations d'analyses que des économistes exercent sur leurs propres pratiques et sur les caractéristiques des cadres théoriques établis. Il s'agit bien, ainsi, d'une opération réflexive d'un milieu sur lui-même.

Dans une perspective d'analyse de discours, deux aspects nous semblent devoir être soulignés. D'une part, il y a un arrêt du processus réflexivité critique qui ne démarque pas frontalement les économistes dans une logique d'adhésion/rejet du discours dominant. La fracture passe à l'intérieur même du discours économique, tel qu'il est codifié par les institutions. D'autre part, l'existence de positions critiques, même minoritaires, signifie que le discours codifié est traversé de tensions, qu'il n'est pas parfaitement homogène et qu'il contient en lui-même des potentialités alternatives. Le verrouillage ne peut s'expliquer exclusivement à partir d'une simple analyse du discours mais il renvoie à ses conditions sociales de production et de diffusion.

Dans l'exemple précédent, le dernier état de réflexivité critique conduisait à remettre en cause l'expression "réformes structurelles » et donc à sortir du discours de référence. Pour assurer la consistance sociale et l'efficace d'un discours, l'arrêt de ce processus de réflexivité semble bien essentiel. Plusieurs procédures de protection peuvent être 
utilisées : négation de la critique par des argumentations théoriques, méthodologiques ou empiriques, neutralisation par le cantonnement du débat à strict niveau théorique interne au seul champ scientifique, marginalisation par l'invocation d'un consensus entre économistes.

b) La nécessité de relier mots et cadres théoriques dans une démarche interprétative et l'attention portée aux diverses significations données à une même expression par des publics aux compétences diverses, souligne qu'il convient d'aller au-delà de la simple correspondance entre mots et variables présents dans le wording. Étant donné la richesse des questionnements que l'on peut adresser aux discours, il n'est guère étonnant que les approches disponibles soient multiples, ainsi que les méthodologies utilisées. J. Angermüller (2007) a offert un panorama des analyses en Europe qui permet de saisir, dans une première approche, la diversité des problématiques et des méthodes qui peuvent constituer des ressources pour une économie politique des discours.

La tendance française s'est formée dans un premier temps dans le cadre de la controverse sur le structuralisme des années 60 associant la référence à Saussure avec celle à Lacan pour la critique du sujet parlant et à Althusser pour la critique des idéologies, puis dans un second temps, à partir de la fin des années 70 , elle opère un tournant pragmatique. L'influence althussérienne est partie des travaux de Michel Pêcheux alors que Foucault étudiait les formations discursives. L'influence pragmatique est présente dans les travaux de Dominique Maingueneau et l'orientation interprétative dans ceux de Jacques Guilhaumou (Guilhaumou, 2005, 2006). La tradition d'analyse lexicométrique est très présente.

La tendance anglo-saxonne est liée au pragmatisme américain et à la philosophie analytique anglaise et pour elle le discours correspond à «l'agir langagier dans une situation donnée ». L'analyse des discours critiques de Norman Fairclough (Fairclough, 1995) cherche à identifier la partie idéologique d'un texte, dans une démarche qui est souvent plus proche de la sociologie compréhensive que de la linguistique ;

La tendance allemande liée dans un premier temps à la théorie de l'agir communicationnel d'Habermas (1987) s'est renouvelée à partir des années 90 en intégrant la sociologie de la connaissance à partir de $\mathrm{M}$. Weber mais aussi celle de Berger et Luckmann (1966). Le discours est appréhendé comme le savoir collectif incorporé aux textes et documents d'une société. Les travaux du sociologue allemand Reiner Keller de l'Université d'Augsbourg visent dans une approche d'analyse du discours interprétative à étudier les bases de la compréhension commune, le savoir collectif que les acteurs mobilisent dans les relations sociales (Keller, 2005, 2012).

Ainsi, au-delà d'une approche des discours réduite à une application de la théorie de l'information, il s'agit d'intégrer le discours comme évènement par définition social, lié à l'activité de communication. L'analyse d'Habermas nous rappelle que l'activité communicationnelle s'exprime "dans la capacité du discours orienté vers l'entente à favoriser l'accord " (Habermas, 2001, p. 51) et qu'elle repose sur certains présupposés relatifs au langage. Elle conduit à faire de l'activité communicationnelle une forme de coordination distincte de la coordination stratégique d'acteurs qui est au cœur, en particulier, des analyses économiques. Dans cette perspective, l'utilisation du langage dans une activité communicationnelle visant l'entente se distingue de son utilisation pour la simple transmission de l'information. Cette démarche peut être distinguée de l'approche dominante en économie d'un double point de vue. D'une part la volonté de rompre avec toute idée de sujet qu'il soit individuel ou qu'il corresponde à un macro sujet 
comme l'État conduit à mettre au centre de l'analyse la relation entre les acteurs dans une démarche plus proche des analyses institutionnalistes que d'une théorie fondée sur le choix rationnel. D'autre part l'idée centrale est que la coordination ne peut être obtenue à travers l'interaction d'individus guidés par leur intérêt personnel: « $\mathrm{Si}$, comme je le suppose avec Durkheim et Parsons, les contextes d'interaction ne peuvent pas être stabilisés par la seule action réciproque d'acteurs agissant en fonction de leurs succès, la société doit être intégrée, en dernière instance, par le moyen de l'action communicationnelle» (Habermas, 1997, p. 40).

21 L'économie des conventions peut aussi être rapprochée d'une analyse qui insiste sur les contraintes inhérentes au langage (cf Eymard-Duvernay, 2009, Diaz-Bone R. et L. Thévenot, 2010)). Le modèle des cités par exemple de Boltanski et Thevenot (Boltanski et Thevenot, 1991) correspond à la mise en évidence de plusieurs régimes de justification. À travers un travail sur des sources textuelles diverses, théoriques ou pratiques, qu'elles relèvent des auteurs de la philosophie politique ou de manuels destinés aux entreprises, les auteurs identifient six cités différentes (marchande, opinion, inspirée, civique, domestique et industrielle) mais caractérisées par une structure commune. Cette structure commune apparaît comme une grammaire qui correspond à l'ensemble des aspects qui organisent la justification : un principe d'équivalence c'est-à-dire un critère à partir duquel sont repérées les différentes cités, un état de grandeur qui renvoie à l'échelle de valeur présente dans chacune d'elle, un format d'investissement qui renvoie au sacrifice accepté pour obtenir un bien et qui assure une relation obligation/droit, une épreuve paradigmatique.

Le terme grammaire utilisé est symptomatique d'une analyse attentive aux ressources du langage. Comme l'énonce F. Eymard-Duvernay «l'homo conventionalis calcule et parle» (Eymard-Duvernay, 2009, p. 131). La référence au « langage ordinaire », la traduction qu'il convient d'opérer entre les cités et qui « sont rendues plus aisées si elles peuvent suivre le frayage d'une association déposée dans la langue » (Boltanski et Thevenot, 1991, p. 445). Il n'est alors guère étonnant que, dans le cadre de l'économie des conventions, l'analyse des discours ait trouvé sa place. Ainsi L'analyse du nouveau discours de management a été développée par E. Chiapello dans un travail en collaboration avec l'analyse du discours critique de N. Fairclough (Chiapello et Fairclough, 2002). Dans une démarche différente P. Abecassis et J-P. Domin (Abecassis et Domin, 2011, 2016) ont étudié les discours dans le domaine de la santé et ont distingué, à l'aide d'une analyse lexicographique, plusieurs types de discours: discours libéral traditionnel, libéral renouvelé et discours marchand. Enfin N. Brisset $(2014,2015)$ développe une approche conventionnaliste de la performativité.

Dans le cadre de la théorie de la régulation, l'approfondissement de la théorie de la politique économique par B. Théret (1999) s'est accompagnée d'une insistance sur le rôle joué par les discours: ils favorisent la communication des agents économiques et politiques en forgeant systèmes d'idées et cadre intellectuel communs. Le discours est présenté comme une médiation du symbolique qui joue un rôle comparable à la monnaie (médiation de l'économique) et au droit (médiation du politique).

Les travaux consacrés aux croyances développés par Frédéric Lordon, s'ils n'ont pas débouché explicitement sur des analyses de discours, permettent de percevoir le lieu où pourrait s'élaborer une analyse régulationniste des discours. La volonté de prendre en compte les croyances et représentations et de développer une analyse des "référentiels " s'accompagne d'une démarche voulant dépasser l'opposition entre structuralisme et 
sociologie compréhensive. Ainsi la position suivante pourrait clairement justifier une démarche relevant de l'analyse de discours : "Ainsi la Régulation, qui n'abandonne en rien son inspiration structuraliste n'en est pas moins en mesure de s'ouvrir à la préoccupation herméneutique, c'est-à-dire au travail des interprétations et des significations mais sans tomber, ni dans le subjectivisme philosophique, ni dans celui, scientificisé, d'un mentalisme cognitiviste. Elle est alors bien placée pour rejoindre à sa façon le déplacement des antinomies de l'explication et de la compréhension, ou de l'objectivisme et du subjectivisme, que d'autres ont formalisés avant elle » (Lordon, 1999, p.171). On pourrait ajouter qu'une économie politique du discours permettrait de dépasser l'opposition, faite par Lordon lui-même entre une économie des conventions focalisée sur l'ordre des justifications et une démarche critique soulignant les rapports de pouvoir et de domination dans l'imposition des croyances. C'est précisément l'étude des modalités discursives par lesquelles l'imposition est effectuée qui conduit à mettre en évidence l'importance des expressions mots, argumentations ou justifications par lesquelles se rendent effectives les modalités de la domination. La matérialité même du discours, ses supports physiques aussi bien que les mots et expressions utilisés évitent tout risque de subjectivisme.

\subsection{Les conditions sociales et institutionnelles des discours économiques}

C'est ici qu'intervient une autre dimension de l'analyse des discours, c'est-à-dire sa mise en relation avec "quelque référentiel social, psychologique, historique (...) » (Maingueneau, 1995, p. 6). Plus précisément les discours renvoient à des communautés discursives liées à des réseaux institutionnels (Maingueneau, 1984, Maingueneau et Cossuta, 1995, p. 115).

La permanence d'un discours suppose l'arrêt d'un processus de réflexivité qui est lié à des mécanismes sociaux et institutionnels. L'enjeu étant alors de faire accepter ces processus en les faisant passer pour l'application de règles liées à la délimitation d'une démarche scientifique, en occultant la logique sociale des rapports de pouvoirs au sein du champ. Des procédures de referees des revues aux commissions d'évaluation des candidatures ou des carrières, en passant par les procédures de désignation du bénéficiaire du prix annuel de la Banque de Suède, les règles sociales et institutionnelles reposent sur la croyance en la neutralité des évaluateurs (Lebaron, 2000, p. 247-248). On a pu souligner le rôle des différences de cultures et d'institutions nationales comme déterminant de la forme et du contenu de l'analyse économique (Fourcade, 2009). De même la structure hiérarchisée du champ est plus forte que dans d'autres disciplines : $45 \%$ des auteurs ayant publié dans le Journal of political economy et $57 \%$ de ceux qui ont publié dans le Quarterly Journal of Economics par exemple ont fait leur thèse dans les cinq principaux départements d'économie des Etats-Unis (Fourcade, Ollion, Algan, 2015, p. 98). Un milieu structuré avec des normes fortes permet de diffuser des manières d'agir et de sentir communes. Ces habitus économistes (Lebaron, 2009) associées à la montée en puissance des économistes néoclassiques font de l'économie, au-delà de spécificités nationales maintenues, une discipline fortement soumise aux normes états-uniennes. Ainsi les économistes peuvent être considérés comme des spécialistes du marché (Lebaron, 2009) ou encore l'« économie-discipline » délimitée comme « l'ensemble des activités qui concourent à la production d'agents calculateurs » (Callon et Latour, 1997). Ce qui conduit légitimement à 
s'interroger sur la responsabilité du discours économique dominant en ce qui concerne le déclenchement de la crise de 2008 (Théret, 2011)2.

Les discours économiques dépendent donc de contextes spécifiques qu'il convient d'identifier. Cette analyse des contextes d'énonciation est ainsi une dimension nécessaire d'une économie politique des discours.

Ceci est d'autant plus important que l'enjeu de l'analyse des discours tient dans leurs effets sur les variables économiques. Cette question des effets a été abordée en particulier par le courant de la sociologie qui cherche à étudier ce qu'il appelle la "performativité des discours économiques", c'est-à-dire les modalités par lesquelles les discours économiques créent le monde qui correspond à leurs conditions de validité (Muniesa, 2014, McKenzie et alii, 2007, Callon, 2007). Ce sont les « agencements sociotechniques » ou les « dispositifs » qui permettent au discours économique de «performer» le monde : le modèle de Black et Scholes a initié un processus de transformation institutionnel des marchés financiers et a permis ainsi de créer les conditions de son utilisation (McKenzie, $2006,2007)$; la réorganisation de la pêche en Norvège a forgé les instruments permettant un calcul coût-avantages qui a débouché sur la diffusion du calcul économique auprès des pêcheurs dont le comportement s'est alors rapproché de celui des agents maximisateurs supposés par la théorie standard (Holm, 1995, 2001).

Cette approche pragmatique, qui aborde le discours économique par ses effets, conduit à se démarquer d'une attitude critique à l'égard de la théorie économique. Le débat sur l'utilisation du concept de performativité, qui consacre un déplacement de l'attention des caractéristiques des discours vers l'étude de leurs effets, semble susceptible de mettre en perspective une telle position. Ce concept, issu de la philosophie pragmatiste d'Austin, renvoie à des énoncés réalisant des actions dans leur énonciation. Dans la sociologie de la performativité le sens est plus large, englobant toutes les modalités par lesquels les énoncés "performent» ou "énactent» le monde social. L'utilisation du terme performation au lieu de celui de performativité semble enregistrer ce glissement (Ambroise, 2015). En soit le débat est d'un enjeu limité dans la mesure où toute approche peut définir ses concepts fondamentaux de la manière la plus adaptée à ses objectifs. Il a pourtant une signification dans la perspective d'une analyse des effets des théories économiques : il s'agit ici davantage d'analyses des effets des théories économiques que de la performativité des discours. Les travaux se centrent plus sur les processus par lesquels des théories, formules ou modèles conduisent à mettre en place des agencements ou dispositifs qui créent leurs conditions de validité, que sur une étude des caractéristiques linguistiques des discours et de leurs conditions pragmatiques d'efficacité. Il serait alors logique de franchir un pas supplémentaire et reconnaître le rôle du pouvoir et du champ du pouvoir, ainsi que celui des procédures donnant autorité à certains locuteurs (en l'occurrence certains économistes) pour énoncer des normes (Ambroise, 2012, 2015)

L'analyse des discours économiques devrait alors prendre en compte l'avertissement de Bourdieu : «(...) la science sociale doit prendre acte de l'autonomie de la langue, de sa logique spécifique, de ses règles propres de fonctionnement. On ne peut en particulier comprendre les effets symboliques du langage sans prendre en compte le fait, mille fois attesté, que le langage est le premier mécanisme formel dont les capacités génératives sont sans limites (...) Toute les théologies religieuses et toutes les théodicées politiques ont tiré parti du fait que les capacités génératives de la langue peuvent excéder les limites de l'intuition ou de la vérification empirique pour produire des discours formellement 
corrects mais sémantiquement vides. Les rituels représentent la limite de toutes les situations d'imposition où, à travers l'exercice d'une compétence technique qui peut être très imparfaite, s'exerce une compétence sociale, celle du locuteur légitime autorisé à parler et à parler avec autorité (...) », (Bourdieu, 1982, p. 20).

Prendre en compte l'autonomie de la langue et les conditions sociales de la production des discours a alors deux conséquences. D'une part, contrairement à la neutralité théorique affichée par la sociologie de la performativité, l'étude des caractéristiques et limites des discours est une partie intégrante et nécessaire de la démarche. Comme l'a souligné l'exemple de la section précédente autour de la notion de réformes structurelles, les conditions sociales de la production et de la diffusion des discours économiques ne peuvent être dissociés des caractéristiques théoriques et logiques de ceux-ci. La seconde conséquence est liée au champ beaucoup plus large de l'analyse des discours, à savoir au fait que, contrairement à ce que pourraient laisser entendre les remarques précédentes, on ne peut se limiter à considérer les discours savants et plus précisément des discours académiques. L'objet porte en effet sur la production langagière d'un ensemble large d'acteurs: organismes administratifs ou internationaux mais aussi presse et acteurs économiques et sociaux.

Dans cette perspective plus large, l'analyse de discours rencontre la notion d'idéologie. De ce point de vue, l'économie politique ne peut que retrouver les préoccupations qui traversent, comme nous l'avons vu dans la première partie, la plupart des théories des discours. Avec la prise en compte de l'autonomie de la langue et des conditions sociales de production des discours, il est logique que les recherches, quels que soient les courants, rencontrent la notion d'idéologie, production symbolique crée par le langage. Or la question de l'idéologie pose la question du référent, présentée comme rapport à la réalité. Le texte précédent de Bourdieu, évoquant les discours «formellement corrects mais sémantiquement vides » renvoie à ce problème.

L'idéologie ne peut être réduite à une illusion (cf. Labica 1987). Chez Marx lui-même l'idéologie peut parfois apparaître plus comme une déformation de la perception que comme une illusion au sens d'erreur ou de "fausse conscience». Dans l'analyse du fétichisme de la marchandise dans Le Capital il utilise l'image de la perception rétinienne qui est d'autant moins négligeable qu'elle était déjà présente dans l'Idéologie allemande: « Et si, dans toute idéologie, les hommes et leurs rapports nous apparaissent placés la tête en bas, comme dans une camera obscura, ce phénomène découle de leur processus de vie historique, absolument comme le renversement des objets sur la rétine découle de son processus de vie directement physique " (1845-1846, [1977]), p. 51). L'idéologie conserve donc de manière déformée les caractéristiques du référent. Dans Le Capital, on retrouve cette approche, lorsque Marx souligne que la circulation des marchandises donne l'apparence d'un rapport entre choses, masquant le rapport entre hommes que la théorie de la valeur permet de mettre au jour. La marchandise n'est pourtant pas qu'une pure illusion, elle est une déformation des rapports plus profonds. En utilisant la comparaison entre la marchandise et le hiéroglyphe, il en fait un objet à interpréter : «La valeur ne porte donc pas écrit sur le front ce qu'elle est. La valeur transforme au contraire tout produit du travail en hiéroglyphe social. Par la suite, les hommes cherchent à déchiffrer le sens de l'hiéroglyphe, à percer le secret de leur propre produit social, car la détermination des objets d'usage comme valeurs est leur propre production sociale, au même titre que le langage. » (Marx, 1993, p. 85). 
On peut comprendre alors que même les «catégories de l'économie bourgeoise » aient une « validité sociale » relative à leur époque : «Ce sont des formes de pensée qui ont une validité sociale, et donc une objectivité, pour les rapports de production de ce mode de production social historiquement déterminé qu'est la production marchande " (Marx, 1993, p. 87). L'activité d'interprétation est ici encore nécessaire pour dégager ce qui constitue une déformation du référent.

Cela conduit à s'interroger sur ce qui relie les énoncés des discours : sont-ils intégrés de manière cohérente (logique) ou sont-ils structurés de manière relâchée autour de quelques notions clés. On peut remarquer par exemple que Bourdieu et Boltanski refusent d'imposer une cohérence à un discours dominant :

Le seul fait de substituer une chaîne d'arguments hiérarchisés et organisés selon un ordre d'allure déductive à la succession souvent anarchique des thèmes et des variations ferait disparaitre ce qui caractérise en propre ces produits pratiquement systématiques de l'application de schèmes générateurs (Bourdieu et Boltanski (1973 [2008,]), p. 10.

Analyser les caractéristiques d'un discours signifie alors être attentif à tous les éléments d'hétérogénéité de décalage et de contradictions internes. On peut sans doute délimiter les contours du discours néolibéral mais il ne faut pas lui attribuer une unité et une cohérence d'ensemble. Les conditions de sa diffusion dépendent d'ailleurs sans doute de cette hétérogénéité qui permet de s'adresser à des publics divers. Comme les discours savants, les discours pratiques sont traversés de contradictions qu'il convient d'identifier. C'est ce que mettent en évidence les situations de crise qui portent en elles les germes de remise en cause et de recomposition.

\section{La crise, moment critique des discours économiques}

37 Un discours économique dominant peut être stabilisé si a) le contenu des énoncés qui le constitue apparait compatible avec les principaux faits économiques auxquels il se réfère. Cette compatibilité est assurée par une interprétation des faits socialement validée ; b) il repose sur un dispositif social et institutionnel.

La crise de 2008 en ébranlant certaines croyances s'est traduite par un certain nombre d'inflexions des discours économiques. Elle nous permet d'appréhender par quels ressorts se structurent et se réorganisent des discours que ce soit dans le champ politicomédiatique (2.1) ou dans le cadre plus spécifique de discours d'organismes internationaux et en particulier de la banque centrale européenne (2.2).

\subsection{Discours politiques, discours économiques : perception et effets des crises financières.}

Ph. Légé et J. Marques Pereira (2013) étudient les caractéristiques et les évolutions des discours économiques tels qu'ils ont été tenus après la crise de 2008. Ils s'intéressent aux discours et entretiens avec la presse des ministres des finances allemands et français, des directeurs successifs de la BCE, aux notes des grandes banques et à certains articles de presse. Il s'agit d'interroger diverses manifestations de la "parole autorisée », pour 
reprendre l'expression de P. Bourdieu. Leur analyse permet d'identifier les différentes périodes de ce qui apparaît comme un moment de crise des discours économique.

Cette période est riche d'enseignement, en particulier en ce qui concerne le rapport discours-faits. Celui-ci ne peut être considéré comme la simple transcription en mots et phrases d'une réalité supposée objective. Comme nous l'avons souligné dans la première partie, un discours est considéré comme une action qui est partie intégrante des phénomènes économiques. Un discours se réfère donc directement ou indirectement, implicitement ou explicitement, à d'autres discours.

41 De ce point de vue, l'étude des différentes sources permet de souligner que, dans une première période, l'importance de la crise fut niée. C'est ce que révèlent en particulier les propos de Christine Lagarde, à l'époque ministre française des finances, qui se voulait rassurante sur la croissance européenne et sur les risques selon elle limités de contagion de la crise américaine. Prendre une perspective d'analyse du discours signifie accorder une importance limitée aux motivations individuelles des locuteurs. Ce qui importe est la fonction que remplit le discours: tenter d'avoir un effet sur les comportements des acteurs et sur leurs motivations. La gravité de la crise bancaire impose alors une inflexion du discours car celui tenu précédemment n'a plus de prise sur la situation nouvelle. Suit alors une période pendant laquelle le couple normalité/urgence permet de justifier des mesures nouvelles en contradiction avec le discours précèdent (nationalisations de certaines banques, déficit budgétaire massif etc.) tout en conservant les caractéristiques fondamentales du discours dominant: la politique monétaire doit viser la stabilisation des prix, la politique budgétaire ne peut être expansionniste et doit viser l'équilibre ou tout au moins un déficit sévèrement limité par des règles énoncées publiquement et des réformes structurelles doivent améliorer la flexibilité des marchés pour accroître le taux de croissance à long terme.

Ce discours dominant est complété par une thématique spécifiquement européenne : celle de la convergence ou non des économies. Ce thème avait à l'origine une fonction précise dans le discours économique en Europe : il fonctionnait comme ce que nous pourrions appeler une prévision légitimatrice. Une prévision car il constituait une affirmation sur l'avenir, légitimatrice car il était un élément d'une argumentation justifiant l'adoption de l'Euro. En tant que prévision, il s'exposait cependant à être démenti par les faits. Tout dépend cependant de l'interprétation des faits, ce qui montre une nouvelle fois que les discours renvoient à d'autres discours. Un premier temps dans l'évolution du discours consistait à avancer l'idée de critères endogènes des zones monétaires optimales : l'évolution économique provoquée par la création de l'Euro créerait les conditions de son succès. Sous l'effet de la crise, une inflexion nouvelle du discours s'est opérée à partir de l'activation d'une opposition centre-périphérie, déjà présente épisodiquement dans la presse, et avec un sens différent, depuis plusieurs années. Ce changement apparaît comme le plus susceptible de maintenir les caractéristiques fondamentales du discours dominant: le couple centre-périphérie permet de mettre l'accent sur l'importance des réformes structurelles et de décharger les responsables des politiques monétaires et budgétaires ainsi que la constitution profondément asymétrique de cette zone mark qu'est, de facto, l'espace monétaire constitué par l'Euro.

43 L'analyse des discours des acteurs pendant la crise de 2008 permet ainsi de souligner les expressions et mots clés par lesquels est assuré le maintien des caractéristiques fondamentales des discours existants. Au-delà de l'apparente variation spectaculaire des comportements, l'analyse des discours permet de mettre en évidence la permanence des 
ressorts fondamentaux des discours dominants. On peut ainsi repérer par quels procédés les discours s'adaptent aux changements de l'environnement.

\subsection{Crise de l'euro}

44 avec la production médiatique. C'est ce qu'étudie Thierry Guilbert (ce numéro). Son analyse permet d'identifier les caractéristiques du discours néolibéral tel qu'il se forme, et se reproduit dans la presse. L'analyse du langage est alors première car elle conduit à repérer les procédés qui forment un discours et à en suivre les effets. Ainsi l'analyse de l'expression « crise de l'euro » correspond à la nominalisation de la phrase « l'euro est en crise » et à un figement dont on peut suivre les multiples occurrences dans la presse. Ce figement a sa logique propre dont l'un est d'incorporer une dimension sacrée c'est-à-dire renvoyant à un élément indiscutable et indiscuté. Reprenant d'O. Reboul la distinction entre sacré montré et sacré caché, Th. Guilbert souligne comment les locutions expriment, parfois malgré elles, ce qui relève de valeurs indiscutables : l'euro et l'Europe apparaissent comme indépassables et la dette comme faute morale, exigeant la discipline des peuples.

analyse linguistique de la presse est particulièrement significative pour l'économiste. D'une part le discours néolibéral a une unité qui tient aux caractéristiques mêmes du langage utilisé dans la presse. Cette unité ne signifie pas que la diversité de contenu est occultée. Il s'agit plutôt d'une différence de perspective que l'on peut penser à travers la distinction entre producteurs de discours et utilisateurs de discours. Ainsi, du point de vue des producteurs le libéralisme contemporain est-il divers, voire profondément divisé. Entre les anarcho-capitalistes tenant d'une défense inconditionnelle de la propriété privée et les ordo-libéraux soulignant la dimension sociale des droits de propriété il n'y a pas de différence de degré mais de nature. Il en est de même du point de vue monétaire de l'opposition entre centralisation de l'offre de monnaie par Friedman et la défense du free banking par Selgin et White. Ces contradictions qui renvoient à la façon même de promouvoir la défense du marché n'entrent pas dans le discours néolibéral, dont le ressort apparait alors ne pas relever d'une défense doctrinale mais de la simple défense de la « réalité ». Une ontologie réaliste sous-tend alors ces discours et est au fondement de son unification.

De la même manière le discours néolibéral entre en relation complexe avec les positions des économistes de la Doxa. On peut identifier ces derniers: une approche positiviste considérant que l'économie est une production fondée sur la construction de modèles théoriques et/ou économétriques validant les énoncés par la cohérence logique des modèles et la confrontation aux faits. Les énoncés obtenus sont considérés comme de nature comparable à ceux des sciences exactes. Cela se traduit, d'un point de vue institutionnel, par une production qui doit passer principalement par des revues scientifiques reconnues. On peut alors aboutir à une situation dans laquelle l'ensemble des énoncés issus de la production d'un économiste ou d'un groupe d'économiste peut être décomposé entre un sous-ensemble compatible avec le discours néolibéral et un second sous ensemble qui s'en différencie. Dans le cadre de la théorie de la croissance endogène, par exemple, la concurrence a des vertus positives pour les pays proches de la frontière technologique mais peut, au contraire, entraver l'innovation et le taux de croissance des pays qui en sont éloignés. Sans soute aboutit-on finalement dans le cas de 
la France à recommander des réformes structurelles assez traditionnellement libérales (Aghion, Cette, Cohen, 2015) dans un ouvrage qui est de statut intermédiaire entre la production de discours relevant du champ scientifique et la défense par la presse des discours existants. L'ambivalence des modèles remplit pourtant clairement une fonction : récuser la référence libérale pour présenter un projet de "changement des mentalités » comme le seul résultat d'une expertise incontestable:

D'autres diront que les idées sont empruntées à la boîte à outils néolibérale et par conséquent les rejetteront sous une étiquette infamante. C'est malheureusement trop souvent la règle du jeu dans le débat français (Aghion, Cette, Cohen, 2015, p. 11).

Il est d'ailleurs significatif que l'on retrouve dans cette citation le procédé de la " comparaison dépréciative » dont F. Lebaron avait étudié le fonctionnement (Lebaron, 2013). De même, l'insistance de Th. Guilbert sur la «stratégie du choc » mise en évidence par N. Klein comme procédé pour faire passer des réformes, peut être illustrée par le titre même du premier chapitre et de ses différentes parties ou sous parties : «Un niveau du PIB qui décroche (...) », "Une situation très dégradée des finances publiques ", «Une égalité des chances en baisse continue ». Enfin le titre du $1^{\text {er }}$ chapitre "Enrayer le déclin » entre étrangement en écho avec cet éditorial de Claude Imbert en 2003 «Ce sera, dit-il, « la réforme ou le déclin ... »; Pardi ce Ministre parle d'or!» (cité par Th. Guilbert, 2011, p. 123).

Il ne s'agit pas ici de discuter des fondements d'une telle approche. Il s'agit plutôt de souligner, à travers cet exemple, à la fois la logique spécifique de la production de discours et la connexion entre des discours qui relèvent du champ scientifique et de ceux qui sont repérables dans la presse.

\subsection{Discours d'organisations en crise : BCE et FMI dans la tourmente}

49 L'analyse du fonctionnement des institutions internationales nous renseigne sur les conditions de production et sur les conditions d'efficacité du langage. Comme l'a souligné Maingueneau (2013), les rapports des organisations internationales appartiennent à des genres routiniers, non seulement par la régularité de leur production mais aussi par les caractéristiques des rapports. L'expression « communauté de communication » " permet ainsi de caractériser la compétence discursive des locuteurs en tant qu'ils partagent des règles de communication et des formes d'évaluation des échanges communicatifs (Maingueneau, 2013, p. 177). Ces rapports dont le caractère "lourdement didactique " donne au lecteur « un effet d'évidence et d'ennui, attaché à tout usage élémentaire du langage » (Maingueneau, 2013, p. 176) sont «à la fois le produit et la condition de leur identité et de leur existence»(p.177). Toutefois au-delà des effets des règles contraignantes, les discours des organisations peuvent être rapportés aux caractéristiques de leur histoire et à la façon dont elles ont réagi aux discours critiques qui leur sont adressés. Les exemples de la BCE et du FMI nous semblent, à cet égard, significatifs.

On peut en premier lieu souligner l'importance de l'analyse du fonctionnement interne des organisations. Comme le montre E. Le Heron dans le cadre de la BCE, la compréhension du comportement d'une organisation nécessite de la replacer dans la perspective historique qui lui a donné naissance. De ce point de vue, les trois influences 
principales repérées à savoir l'européisme, le libéralisme anglo-saxon et l'ordolibéralisme peuvent être interprétées comme des types de discours dont on trouve des traces dans la production discursive de la BCE. On pourrait alors considérer que l'analyse en termes de discours permet de dépasser ce qui apparaît comme des oppositions irréductibles lorsque l'on raisonne en termes d'influences exclusives. C'est, par exemple, le cas de la qualification de la BCE comme institution ordolibérale. Cet ordolibéralisme a été contesté par Bibow (2009) et Théret (2013) alors que l'influence ordolibérale est soulignée (2013) et réaffirmée (ce numéro) par Le Heron. Si la position de Théret et Bibow est stimulante pour remettre en cause la qualification, souvent trop rapide, d'une BCE « ordolibérale ", l'analyse en termes de discours nous permet de dépasser cette apparente divergence d'interprétation, en précisant qu'il s'agit principalement d'insister sur la pluralité des types de discours constitutifs de la pratique d'une organisation. Cette pluralité peut rendre compte de la souplesse de discours susceptibles de s'adapter à des auditoires différents. La justification de l'indépendance de la Banque Centrale par exemple peut tout aussi bien renvoyer à la théorie de la crédibilité des nouveaux classiques qu'à une logique ordolibérale. Une même position peut avoir une signification différente selon le type de discours auquel on le rapporte.

51 Outre cette insistance sur les compromis constitutifs d'une organisation, le mode d'exercice du pouvoir importe aussi pour comprendre le type de discours utilisé comme le souligne le texte d'E. Carré. Il insiste sur le rôle du Chairman dans le prolongement de la théorie des comités de politique monétaire et sur la qualification de CPM « individualistecollégial » et " collégial-autocratique ». Un mode de fonctionnement moins collectif sous la présidence de M. Draghi par rapport à celle de Trichet. La nombre de discours sous la présidence de Draghi est plus réduit que sous celle de Trichet et, lorsqu'en 2013 le nombre de discours remonte, cela n'est pas dû à l'activité du chairman mais à celle des autres membres du directoire de la BCE. Comme cela correspond à une période pendant laquelle des mesures monétaires non conventionnelles ont été affirmées et ont conduit à des tensions au sein de la $\mathrm{BCE}$, on pourrait s'interroger sur le lien entre ces conflits réels ou potentiels et ce processus de « déconcentration » des discours.

Cette perspective entre alors en écho avec l'interprétation proposée par F. Lebaron (ce numéro) qui montre que les rapports de force politiques internes à la BCE débouchent sur des modifications des caractéristiques des discours: le relâchement de l'orthodoxie monétaire conduit, en contrepartie, à un durcissement des règles budgétaires afin de permettre un compromis avec les défenseurs allemands de l'orthodoxie. Pendant la période la plus récente, et face à la critique des effets pervers des politiques d'austérité, un relatif assouplissement budgétaire s'accompagne de discours qui mettent plus fortement l'accent sur les réformes de structures, c'est-à-dire sur la flexibilisation des économies et en particulier du marché du travail.

Bien entendu les caractéristiques des discours ne s'expliquent pas uniquement pas les caractéristiques internes des organisations. Elles renvoient aussi au public visé. Celui-ci, loin d'être une unité indifférenciée, doit au contraire être conçu comme un ensemble structuré. Les acteurs visés sont ceux qui sont initiés et plus particulièrement les porteurs de créances (Clevenot, Desmedt, Lorca, ce numéro). Plus généralement le discours de la Banque centrale se manifeste dans des supports divers qui renvoient à des publics différents : les conférences de presse, les publications de la $\mathrm{BCE}$, le dialogue monétaire de la BCE avec le Parlement européen, (E Carré, ce numéro). De même les discours comme actes de communication ne peuvent qu'évoluer selon les changements qui s'opèrent dans 
les contextes d'action des récepteurs. Si l'on assiste bien à des changements des modalités de communication et des mots utilisés, et si les mots à tonalité négatives l'emportent logiquement au pire moment de la crise financière (Clevenot, Desmedt, Lorca ce numéro), l'ensemble est malgré tout maintenu dans un cadre stable (Lebaron). Ainsi l'étude des discours permet bien d'identifier les facteurs à la fois internes et externes à l'organisation qui déterminent les comportements et leurs effets.

Le cas du FMI illustre les effets des discours critiques sur l'action d'une organisation et sur la recomposition du discours à laquelle ils conduisent. Comme le montrent Fritz et alii (2014), la gestion des crises par le FMI à partir des années 80 a été l'objet de multiples critiques. L'institution a réagi en modifiant ses approches et prescriptions, poussée, sans doute aussi, par la « crise d'identité » qu'a connue l'institution suite aux remboursements de leurs dettes par de nombreuses économies émergentes (Magalhães Prates et Farhi, 2015).

55 Les crises survenues dans des contextes de libéralisation des marchés ne permettaient pas de mettre en cause des politiques macroéconomiques excessivement dépensières (Fritz et al., 2014). Les modèles de seconde génération, avec équilibres multiples et anticipations autoréalisatrices, permettaient de rendre compte de crises déterminées par les processus de marché eux-mêmes, tout en dessinant les contours de l'action à entreprendre. La distinction entre problèmes de liquidité et problèmes de soutenabilité permettait de dessiner plusieurs scénarios envisageables, comme l'indiquait le nouveau document de référence «Prague Framework». Ainsi que le souligne les auteurs, le document suivant "Assessing sustainability" (FMI, 2002) évoquait un flou conceptuel concernant la distinction entre liquidité et soutenabilité. Malgré ce qui apparait comme un flottement sémantique concernant deux mots clés du nouveau discours en construction, cette distinction a continué à jouer un rôle dans l'argumentaire du FMI. C'est donc moins la clarté sémantique qui explique leur utilisation que le rôle qu'ils jouent dans ce discours. En outre, cet exemple montre que la constitution d'un discours trouve son origine davantage dans une logique de confrontation interdiscursive que dans des innovations théoriques issues des développements de la macroéconomie. Ces innovations constituent un ensemble de ressources mobilisables pour des raisons pragmatiques.

Une remarque comparable concerne l'expression "contrôle des capitaux" dont Magalhães Prates et Farhi, (2015) soulignent les formes d'apparition et de disparition dans le discours du FMI. Une nouvelle approche du contrôle des capitaux, montrent-elles, a été introduite, après 2010, en soulignant le caractère temporaire de telles mesures et en réaffirmant la nécessité d'une libéralisation des mouvements de capitaux. Le contrôle des capitaux est l'une des mesures destinées à réguler les mouvements de capitaux et se trouve dans une position soigneusement hiérarchisée: d'abord les politiques macroéconomiques doivent être utilisées et seulement en cas d'inefficacité des mesures plus contraignantes peuvent être prises. Parmi celles-ci il convient alors de distinguer entre réglementation prudentielle du système bancaire et contrôles des capitaux au sens strict qui discriminent entre résidents et non-résidents. Or, comme le montrent Magalhães Prates et Farhi (2015), le critère de discrimination/non-discrimination ne permet pas de distinguer facilement règlementation prudentielle et contrôle des capitaux. Ainsi une distinction entre deux mots, qui ne poserait pas de problème dans le langage ordinaire, est rendu plus difficile dans un discours codifié comme celui du FMI. En outre, cet exemple permet de souligner que la constitution d'un discours d'organisation internationale peut se construire dans une relation implicite avec la 
connotation des mots dans le langage ordinaire. C'est ce que montre, en particulier le destin de l'expression "contrôle des capitaux" remplacée par celle de "mesures de gestion des flux de capitaux » (Magalhães Prates et Farhi, 2015).

De même, la politique promue par le FMI au Brésil peut apparaitre comme la constitution, en actes, d'un contre-discours opposé aux discours critiques concernant son action en Argentine. La position de Barry Eichengreen, défendant le FMI, est de ce point de vue particulièrement significative dans sa formulation même. La politique proposée par l'institution au Brésil offre au Fonds, dit-il, «l'opportunité de démontrer qu'il aide les économies bien gérées comme le Brésil tout en offrant seulement une sévérité bienveillante (tough love ») aux délinquants comme l'Argentine » (Eichengreen, 2002, 3 ; cité par Fritz et alii, 2014, 12).

Cette défense publique est significative par le verbe employé pour désigner l'action du FMI : celle-ci « démontre » le bien fondé des mesures prises. L'action est désignée par un verbe qui renvoie à une argumentation développée dans le langage. Cette action apparaît clairement liée à un discours en construction, discours qui prend la forme d'une argumentation qui repose en partie sur une stigmatisation juridico-morale ("délinquants»). On est ici bien éloigné de la parole officielle apparemment neutre et policée des organisations internationales. C'est ainsi à travers l'attention portée, non pas aux seuls discours officiels mais à une famille de discours exprimant des points de vue complémentaires mais hétérogènes du point de vue de leur expression et de leur tonalité, que l'on peut pleinement saisir les dimensions multiples des expressions associées aux actions menées.

\section{Conclusion}

Les quelques éléments rassemblés ici ne font qu'ébaucher les voies diverses que pourrait emprunter une économie politique des discours. Comme nous l'avons souligné, une telle approche permet de dépasser certaines oppositions entre des analyses qui peuvent apparaitre exclusives lorsque l'on raisonne en termes de confrontation de courants et d'influences pratiques de théories. Que des conflits puissent être dépassés témoigne du déplacement du regard que peut introduire l'analyse du discours et non d'une négation des confrontations théoriques.

La critique des discours existants est, comme nous l'avons souligné, une partie intégrante et nécessaire de l'analyse des discours. De ce point de vue, la perspective d'une critique de l'économie politique s'en trouve élargie car, à une critique de l'économie politique fondée sur l'analyse des théories dominantes, s'ajoute une étude des autres formes de production langagière (rapport, notes, articles de presse, discours oraux) et l'analyse précise des relations entre discours hétérogènes.

61 Enfin, l'un des enjeux d'une économie politique des discours réside dans l'éclairage nouveau qu'elle permettrait de porter sur les problèmes liés aux modes de gouvernement des économies et sociétés contemporaines. Les études sur la gouvernementalité, issues des travaux de Foucault $(1978,1979)$, ont constitué des sources d'inspiration pour des recherches, en particulier en sciences politiques, avec le développement de recherches portant sur le gouvernement à distance par les instruments (Lascoumes et Le Gallès, 2004, Halpern, Lascoumes et Le Galès, 2014). A la question de la formation des instruments et de leurs effets, l'économie politique des discours pourrait ajouter une analyse des modalités 
par lesquelles les croyances économiques influent sur les modes de gouvernementalité. On retrouverait alors, sous un angle renouvelé, la question des « régimes de véridicité », associés, pour Foucault, à l'idée de gouvernementalité.

\section{BIBLIOGRAPHIE}

Abecassis P., Domin J.-P. (2011), « Comment les omnipraticiens expliquent-ils leurs pratiques tarifaires : quelques paradoxes de la politique de santé ", Politiques et management public, vol. XXVIII, $\mathrm{n}^{\circ} 1$, p. 27-55.

Abecassis P., Domin J.-P. (2016), « Le rôle des syndicats de médecins dans la production des idées en matière de politique de conventionnement. Une étude lexicométrique (1971-2008)», Mots, les langages du politique, à paraître.

Ambroise B., (2015) « Performativité de l'économie ou pouvoir symbolique? ", Revue Française de Socio-Économie, 2015/2 (Hors-série), p. 19-35.

Ambroise B. (2012), « Bourdieu et le langage : une théorie de la pratique et du pouvoir linguistiques », in F. Lebaron, G. Mauger (dir.), Lectures de P. Bourdieu, Ellipses, Paris, p. 193-206.

Angermüller, J. (2007), « L'analyse du discours en Europe » In : S. Bonnafous et M.Temmar (dir.), L'analyse du discours en sciences humaines. Paris : Ophrys, p. 9-23.

Austin J. L. (1970), Quand dire c'est faire, Seuil, Paris.

Berger, P. et Luckmann, T. (1966, [2006]), La construction sociale de la réalité, Paris : Armand Colin.

Bibow J. (2009), « On the origin and rise of central bank independence in West Germany », European Journal of History of Economic Thought, 16(1), p. 155-190.

Blinder A. S, Ehrmann M., Fratzscher M., J. De Haan, and D.-J. Jansen (2008) “Central Bank Communication and Monetary Policy: A Survey of Theory and Evidence », Journal of Economic Literature, 46:4, 910-945

Boltanski, L. et Thevenot, L. (1991), De la justification. Les économies de la grandeur, Paris : Gallimard. Bourdieu P. et L. Boltanski (1973 [2008,]) La production de l'idéologie dominante, Paris, Demopolis, Raison d'agir

Bourdieu P. (1982), Ce que parler veut dire. L'économie des échanges linguistiques, Paris : Fayard.

Brisset N. (2012), « Deux approches de l'influence du discours économique sur les phénomènes sociaux », Revue de philosophie économique, 2012/2 vol. 13, pp. 25-62.

Brisset N. (2014), «Performativité des énoncés de la théorie économique : une approche conventionnaliste », thèse de doctorat de l'Université de Lausanne et de l'Université Paris 1 Panthéon Sorbonne.

Callon, M. (2007), « What Does it Mean to Say That Economics Is Performative? », in. D.

MacKenzie, F. Muniesa et L. Siu (2007, dir.), pp. 311-57. 
Callon, M. et B. Latour (1997), “"Tu ne calculeras pas!” ou comment symétriser le don et le capital » In Alain Caillé (sous la direction de) Le capitalisme aujourd'hui, Revue du MAUSS, n 9, La Découverte, Paris, pp. 45-70

Chiapello E. et N. Fairclough (2002) « Understanding the new management ideology: a transdisciplinary contribution from critical discourse analysis and new sociology of capitalism », Discourse \& Society, Vol 13(2): 185-208

Colander D., ed. (1996). Beyond Micro Foundations: Post Walrasian Macroeconomics. Cambridge: Cambridge University Press.

Colander D. (2003) "Post Walrasian Macroeconomics and Heterodoxy Thinking Outside the Heterodox Box", International Journal of Political Economy, vol. 33, no. 2, pp. 68-81.

Eymard-Duvernay F. (2009), « L'économie des conventions entre économie et sociologie : l'homo conventionalis calcule et parle ", in Ph. Steiner, F. Vatin (dir.), Traite de sociologie économique, Presses universitaires de France, Paris, p. 131-164.

Fairclough, N. (1995) Critical Discourse Analysis: The Critical Study of Language. London: Longman. Fourcade, M. (2009), Economists and Societies, Princeton: Princeton University Press.

Fourcade, M. (2015) “The Superiority of Economists”, Journal of Economic Perspective, vol. 29, n.1, p. $89-114$

Guilbert Th. (2011), L'évidence du discours néolibéral. Analyse dans la presse écrite, Bellecombe-enBauges, Éd. Le Croquant, coll. Savoir/agir.

Guilbert Th. (2007) Le discours idéologique ou la force de l'évidence. Paris : L'Harmattan, collection «Logiques sociales».

Guilhaumou, J. (2005) « Où va l'analyse de discours? Autour de la notion de formation discursive », Marges linguistiques 9, pp. 95-113.

Guilhaumou J. (2006), Discours et événement. L'histoire langagière des concepts. Besançon : Presses Universitaires de Franche-Comté.

Habermas J (1987) Théorie de l'agir communicationnel. Tome 1 : Rationalité de l'agir et rationalisation de la société ; Tome 2 : Pour une critique de la raison fonctionnaliste, Paris, Fayard.

Habermas J (1997), Droit et démocratie. Entre faits et normes, Paris, Gallimard, NRF essais

Habermas J (2001), Vérité et justification, Paris, Gallimard, NRF essais

Holm, P. (1995), "The Dynamics of Institutionalization: Transformation Processes in Norwegian Fisheries.” Administrative Science Quarterly 40: 389-422.

Holm, P (2001) The Invisible Revolution: The Construction of Institutional Change in the Fisheries. Norwegian College of Fishery Science, University of Tromsø, Tromsø.

Keller, R. (2005). “Analysing Discourse. An Approach From the Sociology of Knowledge”. Forum Qualitative Sozialforschung / Forum: Qualitative Social Research, 6(3), Art. 32, http://nbnresolving.de/urn:nbn:de:0114-fqs0503327.

Keller, R. 2012. "Entering Discourses: A New Agenda for Qualitative Research and Sociology of Knowledge.” Qualitative Sociology Review 8(2) : 46-75.

Labica G. (1987), Le Paradigme du Grand-Hornu, Paris, La Brèche 
Lascoumes P. et P. Le Gallès (dir.) (2004), Gouverner par les instruments, Paris, Presses de Sciences Po

Halpern C., P. Lascoumes et P. Le Galès (dir.) (2014), L'instrumentation de l'action publique. Controverses, résistance, effets, Paris, Presses de Sciences Po

Lebaron, F. (2000), La croyance économique, les économistes entre science et politique, Paris : Seuil.

Lebaron, F. (2009), « La formation des économistes et l'ordre symbolique marchand », in Ph. Steiner, F. Vatin (dir.), Traite de sociologie économique, Presses universitaires de France, Paris, p. 249-288.

Lebaron, F. (2013), « Pour une sociologie de la production et de la diffusion des discours économiques. Réflexion à partir de l'exemple de la notion de modèle social ", in Temmar M, J. Angermüller, F. Lebaron, (2013) Les discours de l'économie, PUF

Légé Ph. et J. Marques Pereira (2013) « » Les conflits discursifs de gestion de la crise de la dette souveraine dans la zone euro », Colloque du CRIISEA « Discours de la crise et crise du discours ", 5-6 décembre

Lordon F. (1999), «Croyance économique et pouvoir symbolique », L’Année de la régulation, vol. 3 , p. 169-207.

Magalhães Prates D. et M. Farhi, (2015), « From the IMF to the Troika : New analytical Framework, same conditionalities », Economie et institutions, $\mathrm{n}^{\circ} 23$, à paraître

Maingueneau D (1984), Genèses du discours, Liège, Mardaga.

Maingueneau D. (1995) Présentation. In : Langages, $29^{\mathrm{e}}$ année, $\mathrm{n}^{\circ} 117$. Les analyses du discours en France. pp. 5-11.

Maingueneau D., Cossutta F. (1995) «L'analyse des discours constituants », Langages, $29^{\mathrm{e}}$ année, n - 117, pp. 112-125.

Maingueneau D. (2009) Les termes clés de l'analyse du discours, Nouvelle édition, revue et augmentée : collection « Points-Essais », Paris, Seuil, 2009.

Maingueneau D. (2013), « Post-scriptum. Le rapport de la Banque Mondiale. Quelques réflexions d'un analyste du discours », in Temmar M, J. Angermüller, F. Lebaron, (2013) Les discours de l'économie, PUF

Maris Be. (2002), « Introduction », Sciences de la société, $\mathrm{n}^{\circ} 55$.

McCloskey D. N. (1983), « The Rhetorics of Economics », Journal of Economic Literature, volume 31, 1983, p. 482-517

MacKenzie D., F. Muniesa, L. Siu (dir.) (2007), Do Economists Make Markets? On the Performativity of Economics, Princeton University Press

MacKenzie D. (2007), « Is Economics Performative? Option Theory and the Construction of Derivative Markets », in D. MacKenzie, F. Muniesa, L. Siu (dir.)

MacKenzie, D. (2006), An Engine, Not a Camera: How Financial Models Shape Markets. Cambridge, MA : MIT Press. (N.J.), p. 54-86.

Marx K (1993), Le Capital. Livre 1, PUF, 2009, traduction J.-P. Lefebvre.

Marx K. et F. Engels, (1845-1846, [1977]), L'idéologie allemande. 1ere partie Feurbach, Paris Editions sociales, trad. R. Cartelle et G. Badia 
Muniesa F., Callon M. (2009), «La performativité des sciences économiques », in Ph. Steiner, F. Vatin (dir.), Traite de sociologie économique, Presses universitaires de France, Paris, p. 289-324.

Muniesa F. (2014), The Provoked Economy, Economic Reality and the Performative Turn, Routledge, London.

MacKenzie, D. (2003), « An Equation and its Worlds: Bricolage, Exemplars, Disunity and Performativity in Financial Economics », Social Studies of Science, vol. 33, pp. 831-68.

MacKenzie, D. (2004), « The big, bad wolf and the rational market: portfolio insurance, the 1987 crash and the performativity of economics ", Economy and Society, vol. 33, n 3, pp. 303-34.

MacKenzie, D. (2006), «Is Economics Performative? Option Theory and the Construction of Derivatives Markets ", Journal of the History of Economic Thought, vol. 28, n 1, pp. 29-55.

Mirowski, P. et Nik-Khah, E. (2007), « Markets Made Flesh: Performativity, and a problem in Science Studies, Augmentation with Consideration of the FCC Auctions », in. D. MacKenzie, F. Muniesa et L. Siu (2007, dir.), Do Economists Make Markets?, Princeton: Princeton University Press, pp. 190-224.

Temmar M, J. Angermüller, F. Lebaron, (2013) «Introduction. Les discours économiques saisis par les sciences sociales et les sciences du langage ", in : Temmar M, J. Angermüller, F. Lebaron, (2013) Les discours de l'économie, Paris, PUF

Orléan, A. (2002), « Le tournant cognitif en économie », Revue d'économie politique, vol. 112, n 5, pp. 717-38.

Théret B. (1999), « L'effectivité de la politique économique : de l'autopoï̀se des systèmes sociaux à la topologie du social », L’Année de la Régulation, vol. 3, pp. 127-167

Théret B. (2011), « Du keynésianisme au libertarianisme.

La place de la monnaie dans les transformations du savoir économique autorisé », Revue de la régulation [En ligne], 10.

Théret B. (2013), « Dettes et crise de confiance dans l'euro : analyse et voies possibles de sortie par le haut », Revue Française de Socio-Économie, 2013/2 n 12, p. 91-124.

\section{NOTES}

1. Nous parlerons de "théorie dominante ", de théorie néoclassique, voire de « doxa » théorique pour désigner l'ensemble des productions savantes qui revendiquent une scientificité fondée sur un certain nombre de normes : les énoncés valides proviennent soit de la modélisation, soit de la justification statistique et économétrique. En outre la démarche est fondée sur une méthodologie individualiste et l'échange est le mode de coordination principal. Autour de ce noyau normatif, les productions peuvent être diverses : la stricte approche par l'individu maximisateur pouvant être élargie voire dépassée dans le cadre de l'économie comportementale ou de l'économie cognitive ; la coordination marchande pouvant être complétée par la coordination hiérarchique dans les économies des organisations.

2. Notons, d'ailleurs, que B. Théret élargit son interrogation portant sur la responsabilité de la « science économique » à l'ensemble des productions discursives : «Certes la science économique n'est pas seule en cause, car derrière les énoncés de politique économique (budgétaire et monétaire), par-delà "la caution légitimatrice de la science économique ", interviennent également « les prescriptions des grands organismes internationaux, les exigences des marchés financiers, les propos “d'experts”, le tout relayé et consacré par la presse aussi bien financière 
que généraliste qui donne à tous ces discours la plus large audience et joue un rôle de première importance dans l'installation de ces énoncés comme "incontestable" corpus de la politique économique au sein de l'opinion publique» (Lordon, 1999, p. 179). Mais les doctrines économiques sont le point de départ, le cadre cognitif à l'origine de ces diverses prescriptions politiques, exigences financières et propos d'experts » (Théret, 2011).

\section{RÉSUMÉS}

L'article s'interroge sur les conditions permettant d'intégrer l'analyse des discours à l'économie politique. Nous montrons dans un premier temps que l'analyse des discours ne peut être réduite à une correspondance entre mots et variables économiques et doit intégrer une activité d'interprétation. À travers l'exemple de l'économie des conventions et de la théorie de la régulation, nous soulignons que cette analyse des discours est soit déjà présente, même de manière limitée, soit susceptible d'enrichir les analyses existantes Les discours étant considérés comme des activités sociales, la question de leurs conditions de production et de diffusion conduit à s'interroger sur la place des idéologies et sur la façon de les appréhender.

Les moments de crises sont, dans un second temps, abordés comme des moments significatifs dans la mesure où s'y expriment à la fois la volonté d'agir sur le contexte économique par les actes et par les mots et la volonté de maintenir ou de recomposer les discours dominants. Nous discutons en particulier certains travaux qui ont étudié les discours politiques et médiatiques pendant la crise de 2008, la perception de la crise de l'Euro et l'évolution du discours des organisations internationales comme la BCE et le FMI.

The article examines how to integrate discourse analyses into political economy. We show firstly that such analyses cannot be reduced to a correspondence between words and economic variables and must incorporate an interpretive activity. Through the example of the economy of conventions and of the Regulation theory, we emphasize that discourse analysis is either already present, even in a limited way, or is likely to enhance the existing theories. Discourses being considered as social activities, the question of their conditions of production and dissemination raises questions about the role of ideologies.

Secondly, we consider crisis as significant moments, since both express the will to act on the economic situation by actions and words and the desire to maintain or recompose the dominant discourses. We discuss in particular some works that have studied the political and media discourses during the 2008 crisis, the perception of the Euro crisis and the changing discourse of international organizations such as the ECB and the IMF. 
INDEX

Mots-clés : discours, crise, économistes, idéologie, euro, FMI (Fonds monétaire international), BCE (Banque centrale européenne)

Keywords : discourses, crisis, economists, ideology, euro, IMF (International Monetary Fund), ECB (European Central Bank)

Code JEL A11 - Role of Economics • Role of Economists • Market for Economists, A12 - Relation of Economists to Other Disciplines, A14 - Sociology of Economics, B52 - Institutional • Evolutionary, E52 - Monetary Policy, E58 - Central Bank and Their Policies, E02 - Institutions and the Macroeconomy

\section{AUTEURS}

\section{STÉPHANE LONGUET}

Université de Picardie Jules Verne, CRIISEA

JAIME MARQUES PEREIRA

Université de Picardie Jules Verne, CRIISEA 